\title{
Magnetic Configurations and State Diagram of Nanoring Magnetic Tunnel Junctions
}

\author{
Houfang Liu, ${ }^{1}$ Hongxiang Wei, ${ }^{1}$ Xiufeng Han,,${ }^{1,}$ Guoqiang Yu, ${ }^{1}$ Wenshan Zhan,,${ }^{1}$ Sylvain Le Gall, ${ }^{2}$ \\ Yuan Lu, ${ }^{2}$ Michel Hehn, ${ }^{2}$ Stephane Mangin, ${ }^{2, \dagger}$ Mingjuan Sun, ${ }^{3}$ Yaowen Liu, ${ }^{3,+}$ and Cheng Horng ${ }^{4}$ \\ ${ }^{1}$ Beijing National Laboratory of Condensed Matter Physics, Institute of Physics, Chinese Academy of Sciences, \\ Beijing 100190, China \\ ${ }^{2}$ Institut Jean Lamour, UMR 7198, CNRS-Université de Lorraine, BP 70239, 54506 Nancy, France \\ ${ }^{3}$ School of Physics Science and Engineering, Tongji University, Shanghai 200092, China \\ ${ }^{4}$ TDK-Headway Technologies Inc., Milpitas, California 95035, USA
}

(Received 16 November 2016; revised manuscript received 24 July 2018; published 6 November 2018)

\begin{abstract}
Nonvolatile magnetic random-access memory is one of the most promising memory candidates to meet the requirements of high-density and low-power data storage. Downsize scalability and energy efficiency of conventional memory-unit cells with elliptic shape, however, remain matters of great concern. The development of alternative memory-unit-cell architecture that would potentially enhance the performance of practical devices is thus particularly interesting for applications. Here the magnetic configurations for nanoring magnetic tunnel junctions with an in-plane magnetic storage layer are studied by micromagnetic simulation, revealing that, for an appropriate ring width, the outer diameter can be scaled down to less than $20 \mathrm{~nm}$, where the magnetic onion configuration becomes energetically favored. We also study the spin-transfer-torque switching process and dynamic resistance state diagram with respect to the applied magnetic field and spin-polarized current. A low switching-current density is demonstrated. The results indicate the advantage of using nanoring magnetic tunnel junctions as memory units, which may provide an alternative solution for high-storage-density and low-power-consumption nonvolatile memory.
\end{abstract}

DOI: 10.1103/PhysRevApplied.10.054013

\section{INTRODUCTION}

Since 1975, microelectronics has been following Moore's law, which states that the density and speed of integrated circuits will double every 18 months. However, this trend is presently coming to an end, due to insurmountable physical limits and high power dissipation of CMOS. Besides, the next generation of portable, flexible, and wearable electronics devices, especially in the growing Internet-of-things field, are gradually demanding lower-power-consumption and higher-storage-density devices $[1,2]$. The use of devices based on nonvolatile magnetic random-access memory (MRAM), which can significantly reduce the total energy and be integrated into a system on chip without alteration of baseline logic platforms both in process and in design [3-8], has been considered as a promising solution to reduce power consumption, improve reliability, and offer new functionalities. Spintransfer-torque (STT) MRAM with elliptic unit cells with

\footnotetext{
*xfhan@iphy.ac.cn

${ }^{\dagger}$ stephane.mangin@univ-lorraine.fr

†yaowen@tongji.edu.cn
}

an in-plane magnetic anisotropy or circular unit cells with a perpendicular magnetic anisotropy (PMA) has been extensively explored [9-12]. However, low thermal stability and high switching-current density at reduced dimensions still limit the reliability and high density of MRAM. Alternatively, nanoring-shaped magnetic-tunnel-junction (MTJ) cells with closure magnetic flux and circular Oersted field can enable more reliable and lower power consumption [13-16]. Here the magnetic configurations for the free layer of a nanoring MTJ are studied by micromagnetic simulation, revealing an energetically stable onion configuration even with the size scaled down to less than $20 \mathrm{~nm}$ for the outer diameter and $0.5 \mathrm{~nm}$ for the ring width. The switching properties and dynamic resistance state diagram for nanoring MTJs are also been explored. A low switching current of $1.3 \times 10^{6} \mathrm{~A} / \mathrm{cm}^{2}$ is experimentally demonstrated in MTJs with tunnel magnetoresistance ratio of $100 \%$. The nanoring MTJ exhibits the attractive capability of achieving ultralow writing energy (less than $0.4 \mathrm{pJ}$ per bit with a pulse width of $10 \mathrm{~ns}$ ) and high storage density (up to $10 \mathrm{~Gb} / \mathrm{in}^{2}{ }^{2}$. These results provide insights for the further understanding of the magnetization-switching mechanism in nanoring MTJs and offer a design rule for MRAM unit cells with high density and low power consumption. 


\section{METHODS}

Magnetic multilayers with a core structure of buffer/layer $/ \mathrm{IrMn}(10 \mathrm{~nm}) / \mathrm{Co}_{75} \mathrm{Fe}_{25}(2.5 \mathrm{~nm}) / \mathrm{Ru}(0.8 \mathrm{~nm}) /$ $\mathrm{Co}_{40} \mathrm{Fe}_{40} \mathrm{~B}_{20}(3 \mathrm{~nm}) / \mathrm{Mg}(0.4 \mathrm{~nm}) / \mathrm{MgO}(0.6 \mathrm{~nm}) / \mathrm{Co}_{40} \mathrm{Fe}_{40}$ $\mathrm{B}_{20}(2 \mathrm{~nm}) / \mathrm{Ta}(5 \mathrm{~nm}) / \mathrm{Ru}(6 \mathrm{~nm})$ are deposited onto thermally oxidized $\mathrm{Si}$ wafers by dc and $\mathrm{rf}$ magnetron sputtering, and then annealed at $330^{\circ} \mathrm{C}$ in a vacuum under an in-plane magnetic field for $1 \mathrm{~h}$. The bottom, 3-nmthick $(\mathrm{Co}, \mathrm{Fe}) \mathrm{B}$ layer is a reference layer, and the upper, 2-nm-thick $(\mathrm{Co}, \mathrm{Fe}) \mathrm{B}$ layer is a free layer. The fabrication processes for MTJs with an outer diameter of $100 \mathrm{~nm}$ and ring width ranging from 25 to $30 \mathrm{~nm}$ are as follows. First, the bottom electrode is fabricated by ultraviolet lithography combined with Ar-ion beam milling. Second, the nanoring-shaped junction area is patterned by electron-beam lithography using a poly(methyl methacrylate) and hydrogen silsesquioxane bilayer resist technique. The junctions are etched by reactive-ion etching and an Ar-ion beam. Third, the nanoring-shaped MTJs with the top resists are then buried by $\mathrm{SiO}_{2}$ deposition. The resists and $\mathrm{SiO}_{2}$ are removed by a lift-off process before the top electrode is deposited and patterned. Finally, a $\mathrm{Cu}(50 \mathrm{~nm}) / \mathrm{Au}(100 \mathrm{~nm})$ bilayer is deposited as the top electrode [17].

Theoretically, the magnetization reversal in nanoring elements can be well described by micromagnetic simulations [18]. In this study, the magnetic configuration and current-induced dynamics in the nanoring MTJs are modeled with the finite-element micromagnetics package TETRAMAG developed by Hertel et al. [19]. The code is based on the Landau-Lifshitz-Gilbert (LLG) equation extended to include the in-plane Slonczewski and fieldlike spin torques $[3,20]$ :

$$
\begin{aligned}
\frac{d \mathbf{M}}{d t}= & -\gamma_{0}\left(\mathbf{M} \times \mathbf{H}_{\mathrm{eff}}\right)+\frac{\alpha}{M_{S}}\left(\mathbf{M} \times \frac{d \mathbf{M}}{d t}\right) \\
& +\frac{a_{J}}{M_{S}} \mathbf{M} \times\left(\mathbf{M} \times \hat{\mathbf{m}}_{\mathbf{p}}\right)+b_{J}\left(\mathbf{M} \times \hat{\mathbf{m}}_{\mathbf{p}}\right),
\end{aligned}
$$

where $\mathbf{M}$ is the local magnetization of the free layer, $\hat{\mathbf{m}}_{p}$ is the unit magnetization vector of the reference layer, and $\mathbf{H}_{\text {eff }}$ is the effective field that contains the exchange, anisotropy, demagnetizing, stray field (caused by the fixed reference layer) and external magnetic fields. The last two terms in Eq. (1) are the in-plane Slonczewski torque and the fieldlike spin torque, respectively; $a_{J}=\hbar \gamma P J /\left[2 e M_{s} d\left(1+\xi^{2}\right)\right]$ and $b_{J}=\xi a_{J}$, where $\hbar$ is the Planck constant, $\gamma$ is the gyromagnetic ratio, $P$ is the spin polarization, $J$ is the electric current density, $e$ is the electron charge, $M_{s}$ is the saturation magnetization, $d$ is the thickness of the free layer, and $\xi=$ $l_{\mathrm{ex}} / l_{\mathrm{sf}}$ is the ratio of the spin decoherence length and spin-flip relaxation length [20]. In this study, the following parameters are used for the free layer [21,22]: the thickness of the free layer is $2 \mathrm{~nm}, M_{s}=1000 \mathrm{emu} / \mathrm{cm}^{3}$, the exchange constant $A=2 \times 10^{-6} \mathrm{erg} / \mathrm{cm}$, the uniaxial anisotropy $K_{u}=3 \times 10^{4} \mathrm{erg} / \mathrm{cm}^{3}, P=0.56$, the damping constant is 0.01 , and $\xi=0.01$. The average size of the tetrahedron mesh cell is around $2 \mathrm{~nm}$ for a nanoring with a diameter of $100 \mathrm{~nm}$. For samples with a very small ring width of $0.5 \mathrm{~nm}$, the mesh size is decreased to $0.2 \mathrm{~nm}$. In principle, for such small samples, the LLG simulation at the atomistic level may be principally more effective [23]. All the simulations are performed at zero temperature.

\section{RESULTS AND DISCUSSION}

\section{A. Magnetic configurations and extended stability for nanoring MTJs}

First, we study the energetically stable magnetic configurations in the nanoring MTJ using the LLG micromagnetic simulations. The results show that the free-layer magnetization in the nanoring MTJ can exhibit three different magnetic textures - the onion, vortex, and antivortex configurations - as shown in Fig. 1(a). Although each of the three magnetic states is energetically stable (corresponding to one of the local minima of the Gibbs free energy), only the onion or vortex magnetic configuration has the global smallest energy, depending on the structure parameters. This can be recognized from the averaged energy density of the magnetic configurations, which include exchange energy, anisotropy energy, demagnetization energy, and stray field energy caused by the magnetic reference layer of the nanoring MTJ (if any). Figure 1(b) shows the dependence of the energy density on the width of the nanoring for the three magnetic configurations, where the thickness of the free layer is $2 \mathrm{~nm}$ and the outer diameter is fixed to be $100 \mathrm{~nm}$. In this simulation, the stray field generated by the reference layer is assumed to be 150 Oe to reproduce the experimental observations. The vortex configuration (with lower energy) is more energetically favorable when the nanoring width is smaller than a critical value $w_{c}$, and the onion configuration is more favorable for width larger than $w_{c}$. In all cases, the antivortex configuration has much higher system energy. The simulations show the magnetic configuration of the nanoring sample with $2 R=100 \mathrm{~nm}$ and $w=30 \mathrm{~nm}$ is an onion state, which is consistent with the experimental observations as discussed below.

Figure 1(c) displays the magnetization phase diagram for the energetically favored configurations of magnetization for different parameters. The black "nanopillar" line indicates the extreme condition (i.e., the nanoring becomes a pillar without a hole). The blue diamonds and red circles are critical boundaries between the magnetic vortex and onion configurations obtained from the simulations with a stray field and without a stray field, respectively. The energetically favorable configuration of either the onion or the vortex configuration is mainly determined by the comparison of the exchange energy and demagnetization energy, 
(a)
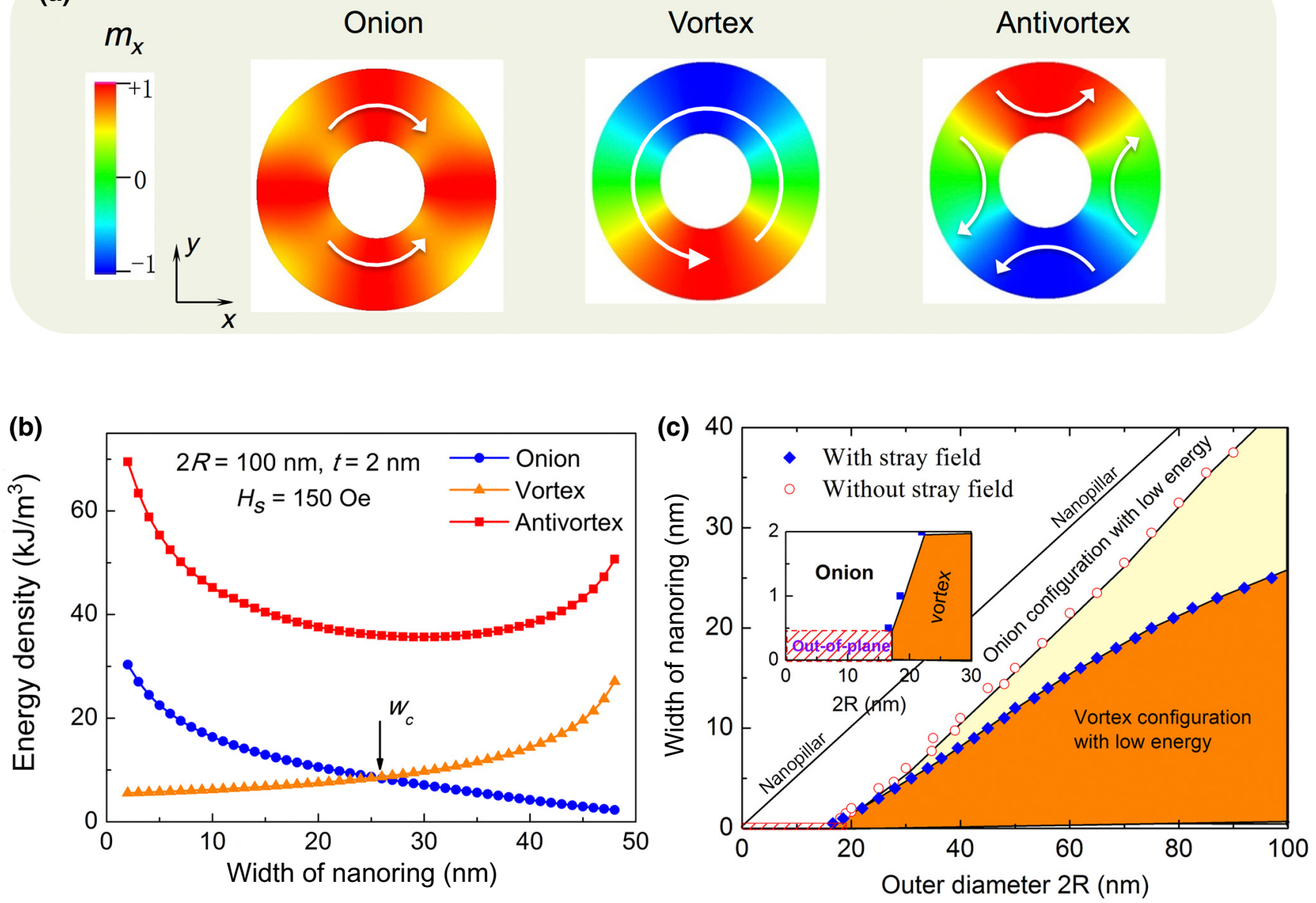

FIG. 1. (a) The appearance of the stable onion, vortex, and antivortex configurations in the free layer of the nanoring MTJs. The magnetization of the reference layer is fixed to the $-x$ direction (i.e., antiparallel alignment for the onion state). (b) Comparison of the energy density as a function of nanoring width simulated at a fixed outer diameter of $100 \mathrm{~nm}$. $w_{c}$ is the critical value of the ring width after which the energy amplitude of the onion configuration is lower than that of the vortex configuration. (c) Calculated magnetization phase diagram in the nanoring MTJs. Here we set the free-layer thickness of the nanorings to be $2 \mathrm{~nm}$. The yellow region is where the vortex (onion) configuration is stable for simulations without (with) the stray field. The inset shows an enlargement for ring width scaling down to $0.5 \mathrm{~nm}$.

as the other energy terms are comparably negligible. The onion configuration is stabler for a ring width greater than the critical value $w_{c}$ [see Fig. 1(b)], at which the inner curvature of the ring leads to increased exchange energy by the misaligned moments of the neighbor cells. The vortex configuration is the favorable texture for a very narrow ring width. Our simulation results indicate that the stable vortex configuration remains for a ring width scaled down to $0.5 \mathrm{~nm}$ while the outer diameter ranges between 20 and $100 \mathrm{~nm}$. Such an ultrathin nanoring structure looks like a spin benzene ring, in which the circular vortex configuration is obviously a stable favorite texture. On the other hand, the stable onion configuration can also be continuously scaled down to smaller size with outer diameter less than $20 \mathrm{~nm}$ and ring width about $0.5 \mathrm{~nm}$ [see the inset in Fig. 1(c)]. Such a phase diagram of magnetic textures should be treated with a lot of caution, especially for practical memory applications at very small lateral sizes. Although the spin vortex is very stable against external perturbations due to its soliton structure with topological protection, the stability factor should still decrease with the downscaling of the in-plane-magnetized free layer due to the relatively low magnetic anisotropy.

Another interesting feature in the inset in Fig. 1(c) is that the nanoring favors the out-of-plane (OOP) magnetization when the ring width is further decreased. This OOP magnetization state is attributed to the shape anisotropy for these geometric parameters, at which the thickness $(2 \mathrm{~nm})$ is larger than the ring width $(0.5 \mathrm{~nm})$. According to Refs. $[9,24,25]$, the $t_{(\mathrm{Co}, \mathrm{Fe}) \mathrm{B}}$ dependence of the effective anisotropy energy density is given by $K=K_{b}+K_{i} / t_{(\mathrm{Co}, \mathrm{Fe}) \mathrm{B}}-\mu_{0} M_{s}^{2} / 2$, where $K_{b}$ is the bulk 


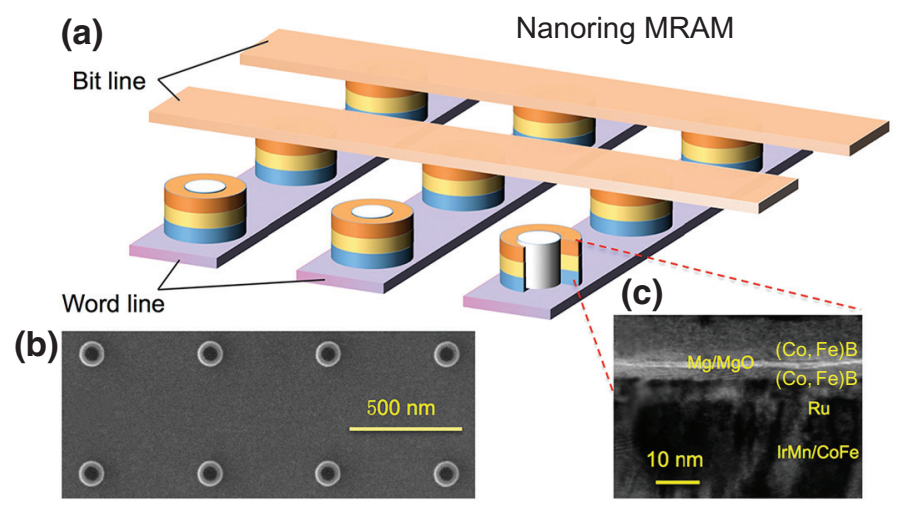

FIG. 2. (a) The architecture for nanoring MRAM containing MTJ cells. Access transistors of MRAM are not shown here. (b) Scanning electron micrograph of nanoring MTJ arrays with an outer diameter of $100 \mathrm{~nm}$ and ring width of $30 \mathrm{~nm}$. (c) Crosssection HRTEM image of the magnetic multilayer film after being annealed at $330^{\circ} \mathrm{C}$ in a vacuum in a magnetic field of $0.8 \mathrm{~T}$. The bottom, 3-nm-thick $(\mathrm{Co}, \mathrm{Fe}) \mathrm{B}$ layer is a reference layer. The upper, 2-nm-thick (Co, Fe)B layer is the free layer, whose magnetization can be switched by application of a magnetic field and injection of polarized current.

crystalline anisotropy, $K_{i}$ is the interfacial PMA between $\mathrm{MgO} /(\mathrm{Co}, \mathrm{Fe}) \mathrm{B}$ interfaces, and the third term is the demagnetization energy. The interfacial PMA is enhanced with the decrease of the free-layer thickness [24]. This will lead to the OOP magnetic texture becoming favorable. Our simulations indicate that the region of the OOP magnetic configuration is significantly enlarged when the free layer thickness, $t_{(\mathrm{Co}, \mathrm{Fe}) \mathrm{B}}$, decreases to $0.5-1.5 \mathrm{~nm}$ and the interfacial PMA is considered (not shown).

\section{B. Dynamic resistance state diagram in nanoring MTJs}

As a magnetic onion state is preferred for nanoringshaped MTJs at the dimensions given, we next systematically study the current-driven magnetic dynamics in MTJs hosting an onion state. To make the onion state favored, we fabricate nanoring-shaped MTJs with outer diameter of $100 \mathrm{~nm}$ and ring width ranging from 25 to $30 \mathrm{~nm}$. From Fig. 1(c), the onion configuration appears in the case of the stray-field effect. The architecture for the nanoring MRAM is shown in Fig. 2(a), with nanoring MTJs as the memory cells. Figure 2(b) shows an array of nanoring MTJs. The thickness of the $\mathrm{MgO}$ barrier is $0.6 \mathrm{~nm}$ to obtain a low resistance-area $(R A)$ product. An ultrathin $\mathrm{Mg}$ layer of $0.4 \mathrm{~nm}$ is inserted before deposition of the $\mathrm{MgO}$ barrier to improve the (001) texture of the $\mathrm{MgO}$ barrier and the interface between the $(\mathrm{Co}, \mathrm{Fe}) \mathrm{B}$ layers and the $\mathrm{MgO}$ layer [26]. Figure 2(c) shows a HRTEM image of the multilayer cross section after annealing. The interfaces between the $(\mathrm{Co}, \mathrm{Fe}) \mathrm{B}$ electrodes and the $\mathrm{MgO}$ barrier are sharp and the roughness of the barrier is low [27].

Figure 3 shows the magnetic properties of the multilayer thin film, and $R-H$ major and minor loops of the nanoring MTJ. In Fig. 3(a), the $M-H$ loop of the multilayer film measured at room temperature (RT) shows two clear steps, indicating that the magnetization switching of the
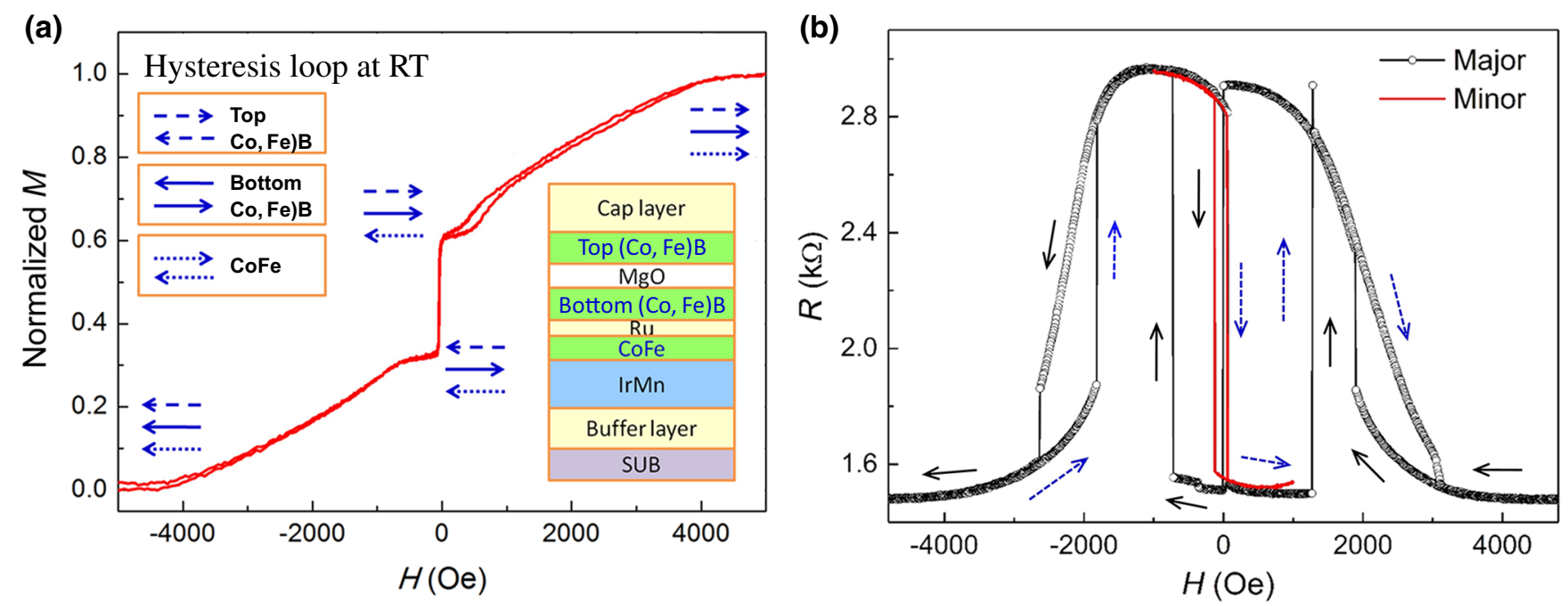

FIG. 3. Magnetic and magnetoelectric transport properties after annealing at $330^{\circ} \mathrm{C}$ with a magnetic field of $0.8 \mathrm{~T}$. (a) $M$ - $H$ loop of the multilayer film measured with a VSM at RT. To show the magnetic-switching processes, three kinds of arrows represent the magnetization direction of the ferromagnetic films: dashed arrows, top (Co, Fe)B layer; solid arrows, bottom (Co, Fe)B layer; dotted arrows, CoFe layer. (b) Major (black line) and minor (red line) $R-H$ loops of the pattered nanoring MgO-based MTJ. The arrows are only guides for the eye. The black arrows indicate the applied magnetic field sweeps from positive to negative, while the blue arrows indicate the applied magnetic field sweeps from negative to positive. The magnetic field is applied in the plane of the multilayer film and the ring. 
(a)
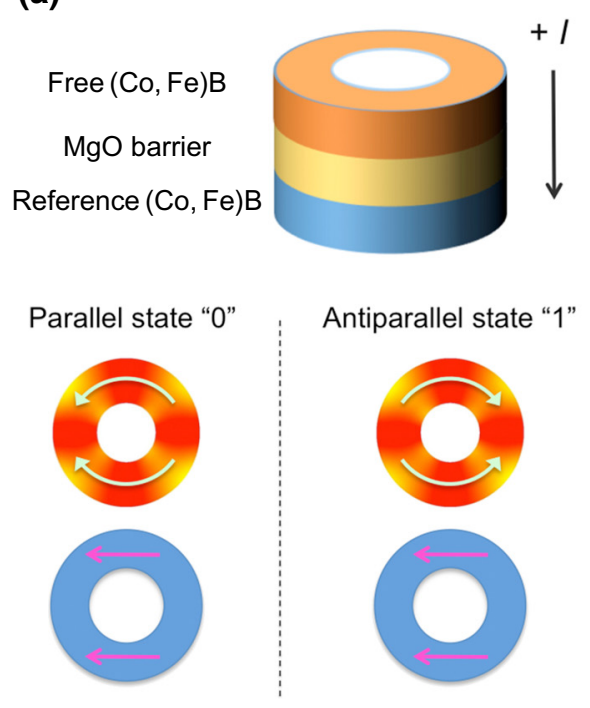

(b)

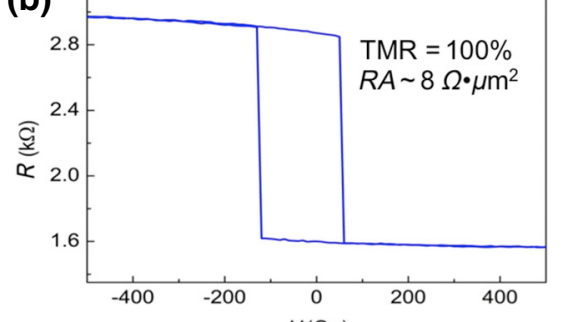

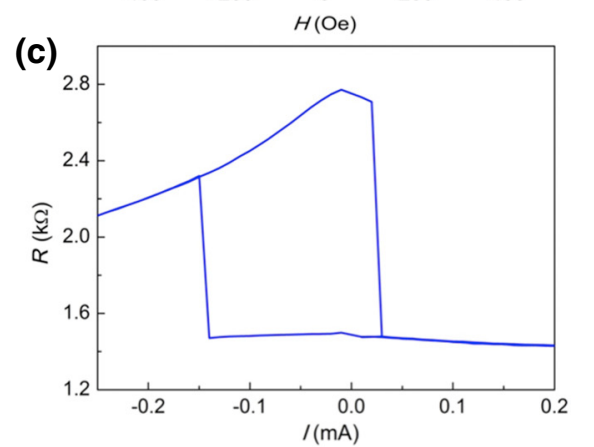

FIG. 4. (a) A nanoring memory cell with positive current from the free layer to the reference layer and magnetization alignment of the two layers: parallel onion state with parallel resistance and antiparallel onion state with antiparallel resistance. (b) Measurements of magnetic-field-driven magnetization switching in a nanoring MTJ at RT. (c) Polarized-current-driven magnetization switching in a nanoring MTJ at RT from the measurements. Positive bias corresponds to electrons flowing from the reference layer to the free layer and thus favors parallel alignment of the two layers. TMR, tunnel magnetoresistance. free and reference $(\mathrm{Co}, \mathrm{Fe}) \mathrm{B}$ layers driven by the magnetic field is clearly observed. The coercivity of the free layer is about $10 \mathrm{Oe}$, which is smaller than that obtained from the $R-H$ loop as shown in Fig. 3(b), probably due to the shape anisotropy or the suppression of domain-structure formation after nanostructure fabrication [24].

The switching properties of nanoring MTJs are shown in Figs. 4(b) and 4(c). Each MTJ is measured by the fourprobe method via leads connected to the top and bottom electrodes. Two types of methods are applied to measure the magnetoelectric transport of the nanoring MTJ: the injection of direct current for spin-transfer torque and small alternating current for the $I-H$ phase diagram using the lock-in technique [28]. The positive bias corresponds to electrons flowing from the reference layer to the free layer and thus favors parallel magnetization alignment of the two layers in Fig. 4(a). Figure 4(b) shows magnetic-fielddriven magnetization switching for the nanoring MTJ. A tunnel magnetoresistance ratio of about $100 \%$ with an $R A$ product lower than $8 \Omega \mu \mathrm{m}^{2}$ is observed at RT. For the nanoring with no current injected, the reversal of the free layer occurs for a coercive field of about 100 Oe, which is significantly larger than the coercive field $(10 \mathrm{Oe})$ of the full film. Figure 4(c) shows current-driven magnetization switching for a nanoring MTJ. The average switching current is defined as $J_{c}=\left(I^{+}-I^{-}\right) / 2 A$, where $I^{+}$and $I^{-}$ are the critical current for the antiparallel-to-parallel magnetization switching and parallel-to-antiparallel switching, respectively, where $A$ is the MTJ cross-section area. In our samples, $J_{c}$ of $1.3 \times 10^{6} \mathrm{~A} / \mathrm{cm}^{2}$ is obtained. Owing to spin excitations at higher bias voltages, the antiparallel resistance $\left(R_{\mathrm{AP}}\right)$ decreases with increasing current $I^{-}$ [29]. The center of the $R-I$ loop is shifted about $0.5 \mathrm{~mA}$, which likely results from the different spin-accumulation efficiency with the different current-flowing directions as well as the ferromagnetic coupling between the reference layer and the free layer. In contrast, the asymmetry of the $R-H$ loop is mainly caused by the ferromagnetic coupling.

The impact of the injected spin-polarized currents on magnetic field switching is also studied. The $R-H$ loops are measured for different values of injected currents, as shown in Fig. 5(a). The orange curve (about zero current) shows a central hysteresis loop. The hysteresis loops move to the left for relatively large positive currents, since positive currents favor the parallel configuration. In contrast, for negative currents, which favor the antiparallel configuration, the hysteresis loops move to the right. With an injected current of $I=-0.15 \mathrm{~mA}$, an abnormal switching-back phenomenon (green curve) is observed with parallel-to-antiparallel and antiparallel-toparallel switching coexisting, as in nanopillar MgO-based MTJs [30]. For a large current $I=-0.25 \mathrm{~mA}$, the purple curve is even farther to the right, with its entire hysteresis outside both the orange curve and the green curve. On the other hand, the $R-H$ curves shift to the left for positive injected current. Similarly, the magnetic fields also have an impact on the current-driven magnetization switching, shown in Fig. 5(b). With fields decreasing from 200 to $50 \mathrm{Oe}$, the $R-I$ loop (blue curve) shifts to the right with a wider hysteresis. A negative field makes the configuration favor the antiparallel state and prevents a positive current reversing the magnetization. When the fields are equal to -50 and -200 Oe, the $R-I$ loops (orange and purple curves) are further shifted to the right.

A series of $R-H$ loops with different injected currents (or a series of $R-I$ loops at various applied magnetic fields) allow us to plot the experimental state diagram. The difference in resistance $\left[R_{\text {diff }}(H)=R_{\text {inc }}(H)-R_{\text {dec }}(H)\right]$ between the increasing and decreasing parts of the hysteresis loop can be extracted from the $R-H$ loops [28], from which a 

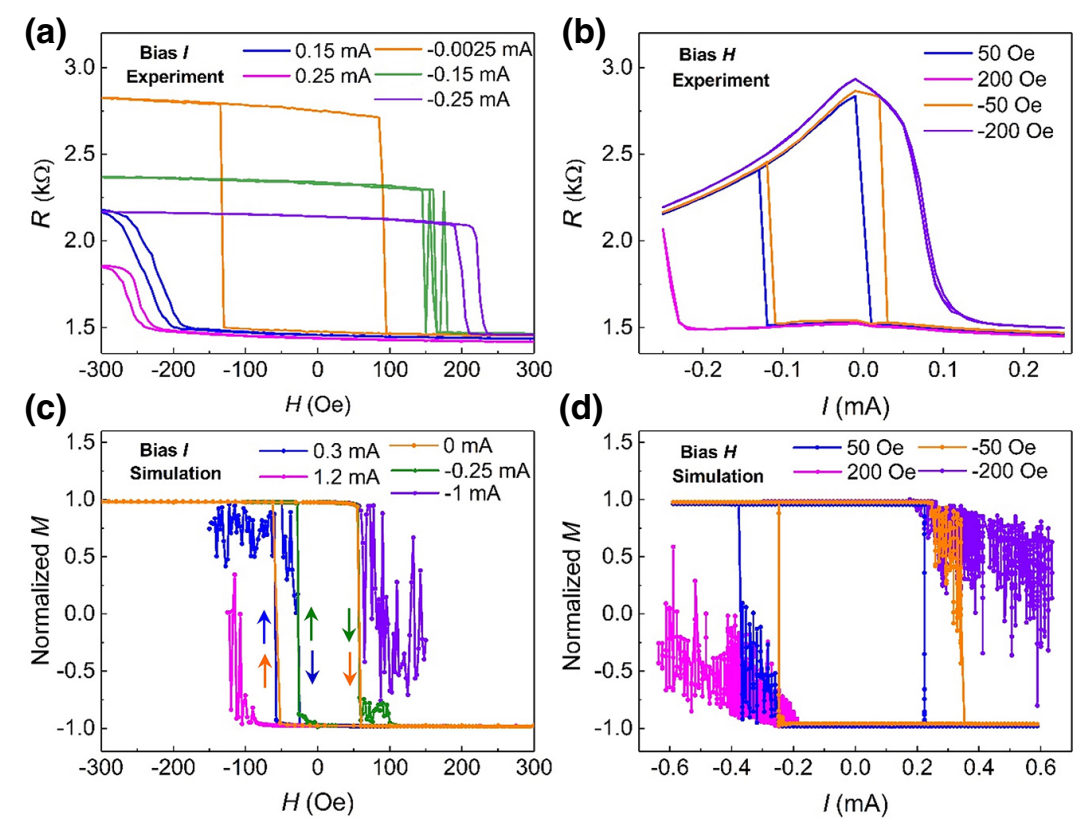

(e)

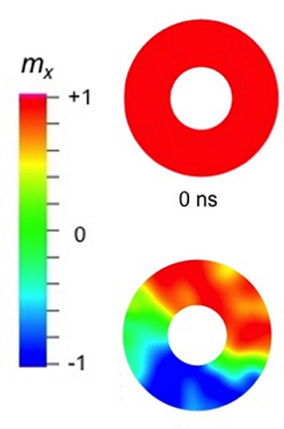

4 ns

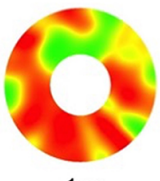

$1 \mathrm{~ns}$

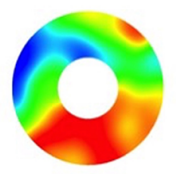

$6 \mathrm{~ns}$

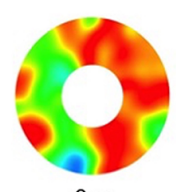

2 ns

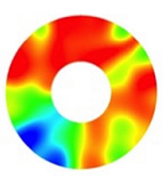

8 ns

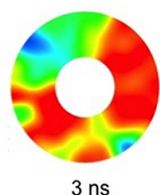

3 ns

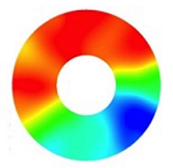

$10 \mathrm{~ns}$

(f)

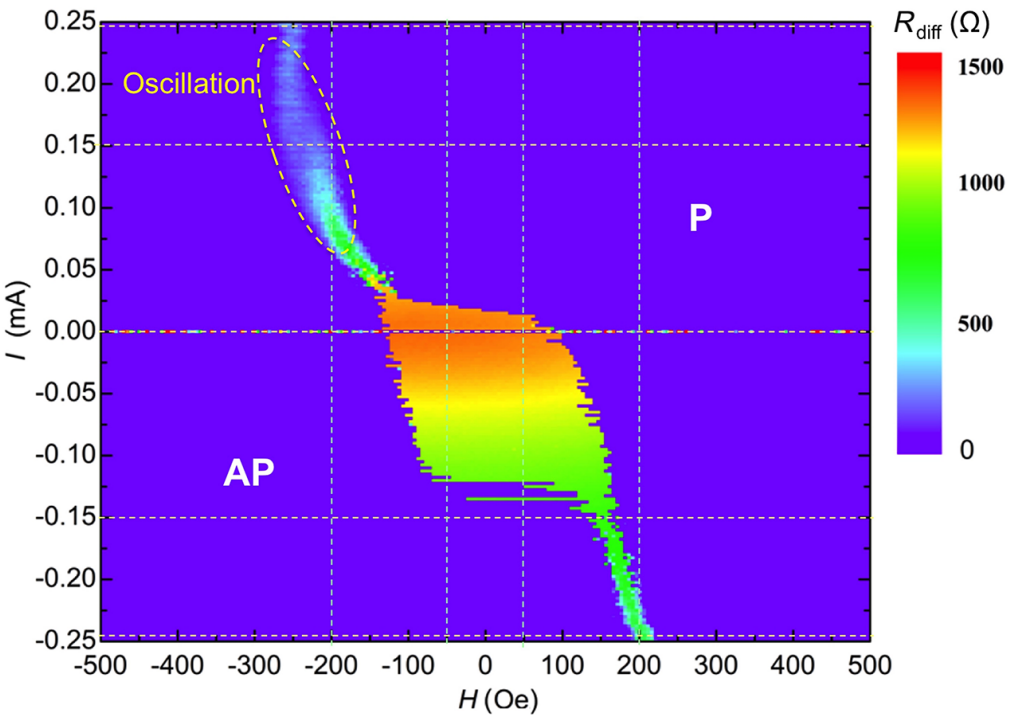

FIG. 5. (a) $R$ - $H$ loops with a series of bias injected currents $I=0.0025 \mathrm{~mA}, \pm 0.15 \mathrm{~mA}$, and $\pm 0.25 \mathrm{~mA}$, respectively. (b) $R$ - $I$ loops with a series of magnetic fields $H= \pm 50$ Oe and \pm 200 Oe, respectively. Corresponding micromagnetic simulation curves based on the LLG equation for normalized magnetization $\left(m_{x}\right)$ varied with the magnetic field and spin-polarized current. (c) $m_{x}-H$ loops with different bias injected currents. For clarity, differently colored arrows denote the corresponding switching curves. (d) $m_{x}-I$ loops with various bias magnetic fields. (e) A series of transient snapshots of free-layer magnetization configurations driven at $H=-200 \mathrm{Oe}$ and $I=0.6 \mathrm{~mA}$. (f) The $I-H$ state diagram of the difference in resistance $R_{\mathrm{diff}}(H)=R_{\mathrm{inc}}(H)-R_{\mathrm{dec}}(H)$. The colored scale bar corresponds to the value of the difference in resistance $R_{\text {diff. }}$ AP, antiparallel; P, parallel. 


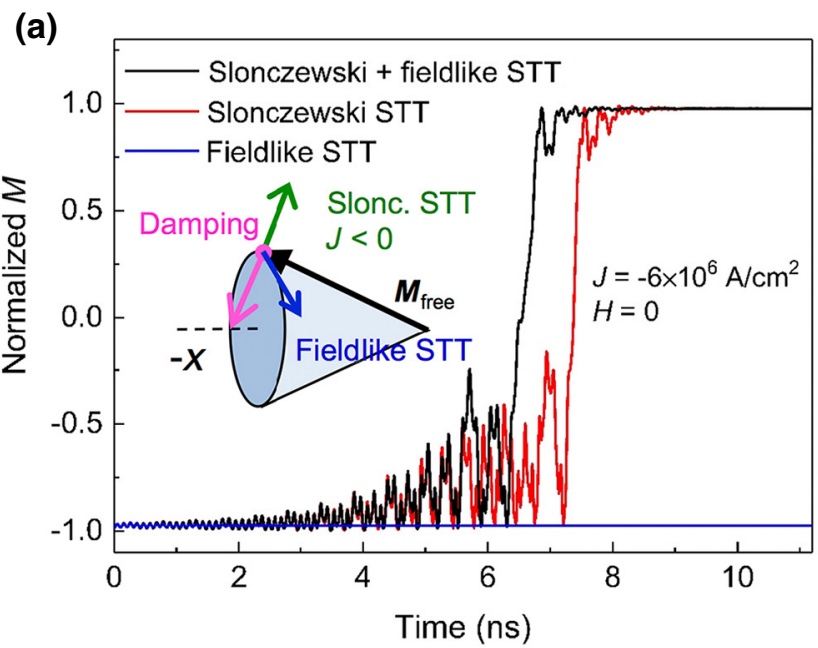

(c)

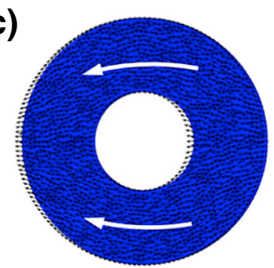

$t=0 \mathrm{~ns}$

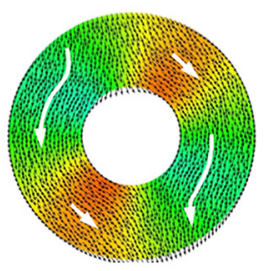

$6.65 \mathrm{~ns}$

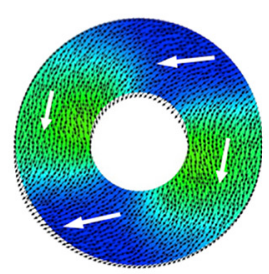

$6.38 \mathrm{~ns}$

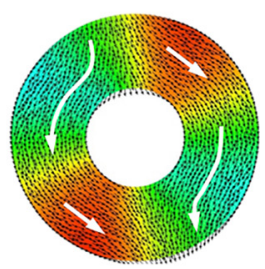

$6.66 \mathrm{~ns}$ (b)
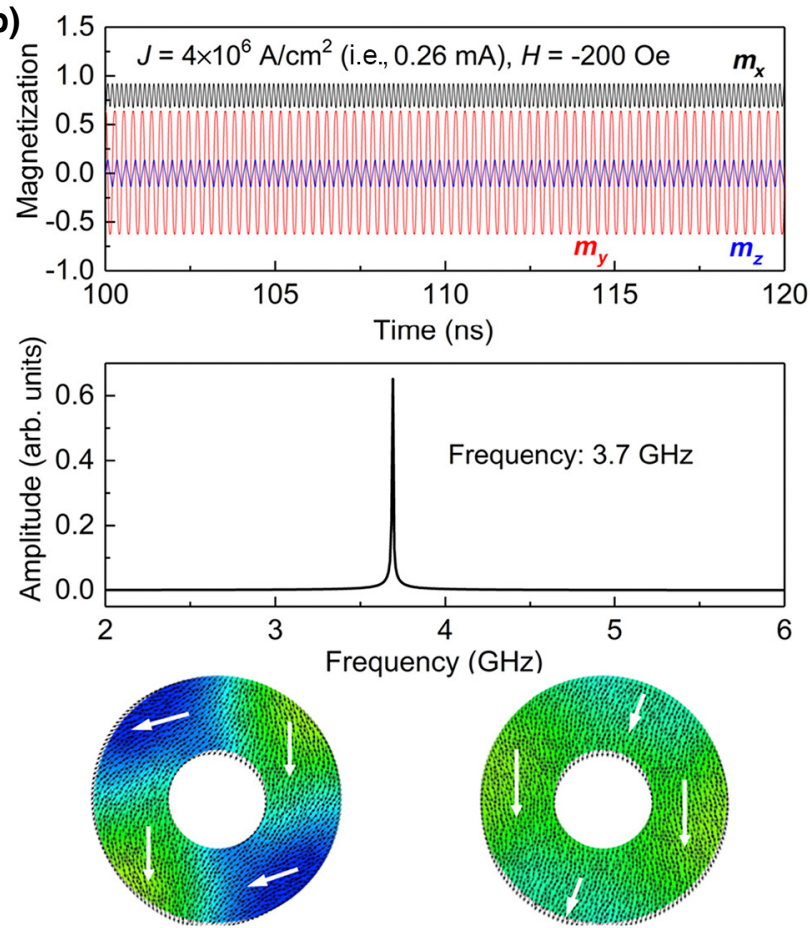

$6.50 \mathrm{~ns}$

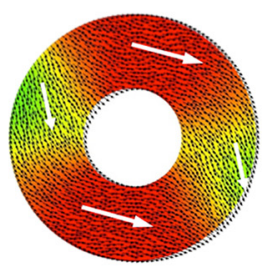

$6.78 \mathrm{~ns}$

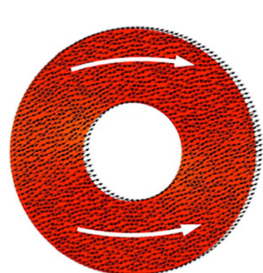

$7.10 \mathrm{~ns}$

FIG. 6. (a) Micromagnetic simulation performed in the switching-parameter region, $J=-6 \times 10^{6} \mathrm{~A} / \mathrm{cm}^{2}$ (i.e., $I=-0.39 \mathrm{~mA}$ ) and $H=0$. Current-induced magnetization switching simulated with the in-plane Slonczewski STT, fieldlike STT, and their combination. The inset shows the orientations of the in-plane Slonczewski STT (green arrow), the field-like STT (blue arrow), and the damping torque (pink arrow). The in-plane Slonczewski STT drives the free layer to switch into the opposite direction, while the fieldlike torque changes the frequency of magnetization precession. (b) Current-driven magnetization oscillations. Normalized magnetization vector ( $\mathbf{m}: m_{x}, m_{y}$, and $m_{z}$ ) as a function of time for magnetization precession with $J=4 \times 10^{6} \mathrm{~A} / \mathrm{cm}^{2}$ and $H=-200$ Oe. (c) The snapshots of the magnetic configuration during the magnetization switching of the free layer in nanoring MTJs.

region of dynamic magnetization states driven by the field or current can be highlighted. Therefore, the state diagram is built as a two-dimensional colored $(I, H)$ map, where each point corresponds to a specific pair of the current and field values. The color of the $(I, H)$ map represents the value of $R_{\text {diff }}$ at these coordinates. Figure 5(f) shows the state diagram of $R_{\text {diff }}$ as a function of the magnetic field $H$ and current $I$, which can be mainly divided into three regions: the parallel, antiparallel, and bistable states. The bistable-state region corresponds to the area where the MTJ can be in either a parallel state or an antiparallel state depending on the history of the device. More interesting, in the zone with $H \sim-250$ Oe and $0.1 \mathrm{~mA}<I<0.25 \mathrm{~mA}$, an area can be observed with $R_{\text {diff }} \neq 0$, which has been identified as current-induced steady-state magnetization precession and spin-transfer nano-oscillators [28]. This feature is discussed further in Fig. 6(b).

\section{Micromagnetic simulation for the state diagram of nanoring MTJs}

To gain insight into the observed state diagram, micromagnetic simulations based on the LLG equation including the current-induced STT effect are performed for the nanoring MTJs. We show the spatiotemporal dynamics of the free layer driven by the combined effect of the STT and an applied magnetic field. In our simulations, both the current-induced Slonczewski STT and the fieldlike STT are considered. The simulated curves indicate the $x$ component of magnetization $\left(m_{x}\right)$ varies with the 
magnetic fields and injected currents as shown in Figs. 5(c) and 5(d), respectively, where the simulations are at each field or current step run for $10 \mathrm{~ns}$. Similarly to the experimental results shown in Figs. 5(a) and 5(b), shifts of the $m_{x}-H$ and $m_{x}-I$ loops are also observed with a series of bias currents and magnetic fields. A central hysteresis loop at zero current is observed (orange curve) in Fig. 5(c). As the negative bias current increases, the switching loop starts to move right. This is caused by the negative-currentinduced STT effect, which drives the free layer to be the antiparallel state. For a large current (e.g., $-1 \mathrm{~mA}$ ), the magnetization-switching curve exhibits strong telegraphnoise-like fluctuation behavior. This telegraphlike noise is obtained at zero temperature, rather than excited by effective temperature. This cannot be explained by the coherent spin-torque model, because the dwell time in one state at a certain current $I$ and magnetic field $H$ is proportional to $\exp \left[E(I, H) / k_{B} \mathrm{~T}\right][31]$, where $E(I, H)$ represents the energy barrier corresponding to available magnetic states. According to the results of Lee et al. [32], this phenomenon can be ascribed to the spatial inhomogeneous magnetization dynamics, which could generate the incoherent local precession frequency and spin-wave excitation. A series of transient snapshots taken from the magnetizationevolution process for $I=0.6 \mathrm{~mA}$ and $H=-200$ Oe [corresponding to the purple curve in Fig. 5(d)] are given in Fig. 5(e), showing the typical spatially inhomogeneous magnetic configuration dynamics. The simulation starts from an antiparallel state $\left(m_{x}=+1\right)$ at $H=-200$ Oe. When a current of $0.6 \mathrm{~mA}$ is applied, the current-induced STT effect tries to drive the free layer to the parallel state $\left(m_{x}=-1\right)$. The competition between the magnetic field and the STT effect, together with the nonuniform local demagnetizing field, results in chaotic inhomogeneous magnetic configurations. Consequently, fluctuation magnetization of $m_{x}$ is observed in Fig. 5(d) (purple curve). In contrast, the telegraphlike noise is also observed in the case of large negative currents and positive magnetic field bias [e.g., 200-Oe curve in Fig. 5(d)]. In this case, the current-induced STT tries to drive the free layer to the antiparallel state $\left(m_{x}=+1\right)$ and the bias field tries to drive it to the parallel state $\left(m_{x}=-1\right)$. Similarly, the $M-H$ curves in Fig. 5(c) shift to the left for positive bias current and to the right with negative bias current.

Figure 6(a) shows the simulated current-driven magnetization switching in a $100-\mathrm{nm}$ nanoring, in which we compare the combined STT effect (black curve) with the individual effect of the Slonczewski STT (red curve) and the fieldlike STT (blue curve). The simulations start with an initial parallel state (i.e. $m_{x}=-1$ ) and are performed at a current density of $J=-6 \times 10^{6} \mathrm{~A} / \mathrm{cm}^{2}$ and $H=0$ Oe. The results clearly indicate that the fieldlike STT alone cannot cause magnetization switching. In contrast, the Slonczewski STT alone as well as the combined STT can reproduce the magnetization switching to the antiparallel state $\left(m_{x}=+1\right)$. Additionally, the latter switches a little faster. These simulation results can be easily explained by a torque model as illustrated in the inset in Fig. 6(a). The Slonczewski STT can overcome the damping torque and drive the free layer in the opposite direction, while the fieldlike STT speeds up the magnetization rotating around the effective field. Obviously, the fieldlike STT alone cannot switch the magnetization, as confirmed by the simulation results. The current-induced magnetization switching is mainly determined by the competition between the Slonczewski and Gilbert damping torque. In contrast to metallic or giant-magnetoresistance structures $[33,34]$, an enhanced fieldlike STT with typically $10 \%-30 \%$ of the amplitude of the in-plane Slonczewski spin torque has been reported in MTJs [35,36]. As observed in Fig. 6(a) the fieldlike STT can change the frequency of the magnetization's precession motion. Figure 6(c) shows a series of transient snapshots taken during the magnetization evolution in the case of both the in-plane Slonczewski STT and the fieldlike STT. The free layer starts from an onion configuration, which is a parallel magnetization-alignment state with respective to the reference layer. After the current is applied, the current-induced STT effect breaks the equilibrium, leading to the local moment departure from the $-x$ direction. The magnetization misalignment between the reference layer and the free layer will in turn locally enhance the spin-transfer torque [3]. Thus, two magnetic domains with opposite direction (along the $+x$ axis) nucleate at $t=6.65 \mathrm{~ns}$. After that, the reversed domain expands toward the neighboring parts through the exchange coupling and the STT effect, resulting in more magnetic moments switching to the $+x$ direction. Finally, the free layer is fully switched to the antiparallel state at $t=7.10 \mathrm{~ns}$.

To obtain a better understanding of the magnetizationoscillation state experimentally observed in the top-left and bottom-right precession regions of the state diagram in Fig. 5(f), we further perform a series of simulations. Figure 6(b) shows a typical magnetization precession of a free layer excited at $H=-200$ Oe and $J=4 \times 10^{6} \mathrm{~A} / \mathrm{cm}^{2}$ (i.e., $0.26 \mathrm{~mA}$ ). In this simulation, the initial magnetization starts from an antiparallel alignment (onion configuration) with $\left\langle m_{x}\right\rangle \approx 1$. The STT generated by the positive current tries to drive the free-layer magnetization to the parallel state, while the applied negative magnetic field prevents this rotation. The competition between those terms leads to a state of dynamical equilibrium, where the magnetization undergoes steady-state precession along an approximately elliptic trajectory. The calculated FFT spectrum of the magnetization precession exhibits a microwave frequency of $3.7 \mathrm{GHz}$. With the magnetic field amplitude increasing from -300 to $-400 \mathrm{Oe}$, the oscillation frequency can be tuned from 4.2 to $5.7 \mathrm{GHz}$. Similarly, the oscillation frequency can be tuned by the variation of the current. 


\section{CONCLUSION}

In summary, we theoretically predict the scalability of the static phase diagram of ring-shaped magnetic tunnel junctions with outer diameter of less than $20 \mathrm{~nm}$. Our results show that with appropriate adjustment of the nanoring shape parameters, the onion configuration is the energetically stablest state. The properties of current-driven magnetization switching in $\mathrm{a}(\mathrm{Co}, \mathrm{Fe}) \mathrm{B} / \mathrm{MgO} /(\mathrm{Co}, \mathrm{Fe}) \mathrm{B}$ nanoring MTJ are experimentally reported. A switchingcurrent density as low as $1.3 \times 10^{6} \mathrm{~A} / \mathrm{cm}^{2}$ in a nanoring MTJ with an outer diameter of $100 \mathrm{~nm}$ and narrow ring width between 25 and $30 \mathrm{~nm}$ is experimentally demonstrated. The dynamic resistance state diagram as a function of $I-H$ for the nanoring MTJ is explored, and the findings are supported by micromagnetic simulations. The results provide insights for the further understanding of the magnetization-switching mechanism in nanoring MTJs, offering a design for MRAM with high density and lowpower consumption.

\section{ACKNOWLEDGMENTS}

The project was supported by the National Key R\&D Program Project of the Ministry of Science and Technology (MOST; Grant No. 2017YFA0206200), the MOST National Key Scientific Instrument and Equipment Development Projects (Grant No. 2011YQ120053), the National Natural Science Foundation of China (NSFC; Grants No. 11434014, No. 51229101, No. 11674373, No. 51501098, and No. 51620105004), the Strategic Priority Research Program (B) of the Chinese Academy of Sciences (Grant No. XDB07030200), and an international collaborative research program between NSFC and the French Agence Nationale de la Recherche (ANR; Grant No. F040803). Y.W.L. thanks NSFC for support (Grants No. 11774260 and No. 51471118). Y.L. acknowledges the joint ANRNSFC SISTER project (Grants No. ANR-11-IS10-0001 and NNSFC 61161130527). We thank Professor Zhongming Zeng for discussions.

[1] M. Stoppa and A. Chiolerio, Wearable electronics and smart textiles: A critical review, Sensors 14, 11957 (2014).

[2] Y. Ji, D. F. Zeigler, D. S. Lee, H. Choi, A. K.-Y. Jen, H. C. Ko, and T.-W. Kim, Flexible and twistable non-volatile memory cell array with all-organic one diode-one resistor architecture, Nat. Commun. 4, 2707 (2013).

[3] J. C. Slonczewski, Current-driven excitation of magnetic multilayers, J. Magn. Magn. Mater. 159, L1 (1996).

[4] E. B. Myers, D. C. Ralph, J. A. Katine, R. N. Louie, and R. A. Buhrman, Current-induced switching of domains in magnetic multilayer devices, Science 285, 867 (1999).

[5] S. Mangin, D. Ravelosona, J. A. Katine, M. J. Carey, B. D. Terris, and Eric. E. Fullerton, Current-induced magnetization reversal in nano-pillar with perpendicular anisotropy, Nat. Mater. 5, 210 (2006).

[6] Y. M. Huai, F. Albert, P. Nguyen, M. Pakala, and T. Valet, Observation of spin-transfer switching in deep submicronsized and low-resistance magnetic tunnel junctions, Appl. Phys. Lett. 84, 3118 (2004).

[7] Z. Diao, D. Apalkov, M. Pakala, Y. Ding, A. Panchula, and Y. Huai, Spin transfer switching and spin polarization in magnetic tunnel junctions with $\mathrm{MgO}$ and $\mathrm{AlO}_{\mathrm{x}}$ barriers, Appl. Phys. Lett. 87, 232502 (2005).

[8] H. Kubota, A. Fukushima, Y. Ootani, S. Yuasa, K. Ando, H. Maehara, K. Tsunekawa, D. D. Dyayaprawira, N. Watanabe, and Y. Suzuki, Evaluation of spin-transfer switching in $\mathrm{CoFeB} / \mathrm{MgO} / \mathrm{CoFeB}$ magnetic tunnel junctions, Jpn. J. Appl. Phys. 44, L1237 (2005).

[9] S. Ikeda, H. Sato, M. Yamanouchi, H. Gan, K. Miura, K. Mizunuma, S. Kanai, S. Fukami, F. Matsukura, N. Kasai, and H. Ohno, Recent progress of perpendicular anisotropy magnetic tunnel junctions for nonvolatile VLSI, Spin 2, 1240003 (2012).

[10] A. V. Khvalkovskiy, D. Apalkov, S. Watts, R. Chepulskii, R. S. Beach, A. Ong, X. Tang, A. Driskill-Smith, W. H. Butler, P. B. Visscher, D. Lottis, E. Chen, V. Nikitin, and M. Krounbi, Basic principles of STT-MRAM cell operation in memory arrays, J. Phys. D 46, 074001 (2013).

[11] Z. T. Diao, Z. J. Li, S. Y. Wang, Y. F. Ding, A. Panchula, E. Chen, L.-C. Wang, and Y. M. Huai, Spin-transfer torque switching in magnetic tunnel junctions and spin-transfer torque random access memory, J. Phys.: Condens. Matter 19, 165209 (2007).

[12] D. Apalkov, A. Khvalkovskiy, S. Watts, V. Nikitin, X. T. Tang, D. Lottis, K. Moon, X. Luo, E. Chen, A. Ong, A. D. Smith, and M. Krounbi, Spin-transfer torque magnetic random access memory (STT-MRAM), J. Emerg. Technol. Comput. Syst. 9, 13 (2013).

[13] F. Q. Zhu, G. W. Chern, O. Tchernyshyov, X. C. Zhu, J. G. Zhu, and C. L. Chien, Magnetic Bistability and Controllable Reversal of Asymmetric Ferromagnetic Nanorings, Phys. Rev. Lett. 96, 027205 (2006).

[14] K. Martents, D. L. Stein, and A. D. Kent, Magnetic reversal in nanoscale ferromagnetic rings, Phys. Rev. B 73, 054413 (2006).

[15] T. J. Hayward, J. Llandro, R. B. Balsod, J. A. C. Bland, D. Morecroft, F. J. Castaño, and C. A. Ross, Switching behavior of individual pseudo-spin-valve ring structures, Phys. Rev. B 74, 134405 (2006).

[16] H.-X. Wei, J. X. He, Z.-C. Wen, X.-F. Han, W.-S. Zhan, and S. F. Zhang, Effects of current on nanoscale ring-shaped magnetic tunnel junctions, Phys. Rev. B 77, 134432 (2008).

[17] Z. C. Wen, H. X. Wei, and X. F. Han, Patterned nano-ring magnetic tunnel junctions, Appl. Phys. Lett. 91, 122511 (2007).

[18] G. D. Chaves-O'Flynn, A. D. Kent, and D. L. Stein, Micromagnetic study of magnetization reversal in ferromagnetic nano-rings, Phys. Rev. B 79, 184421 (2009).

[19] R. Hertel, W. Wulfhekel, and J. Kirschner, Domain-Wall Induced Phase Shifts in Spin Waves, Phys. Rev. Lett. 93, 257202 (2004).

[20] S. Zhang, P. M. Levy, and A. Fert, Mechanisms of Spin-Polarized Current-Driven Magnetization Switching, Phys. Rev. Lett. 88, 236601 (2002). 
[21] T. Devolder, L. Bianchini, K. Miura, K. Ito, Joo-Von Kim, P. Crozat, V. Morin, A. Helmer, C. Chappert, S. Ikeda, and H. Ohno, Spin-torque switching window, thermal stability, and material parameters of $\mathrm{MgO}$ tunnel junctions, Appl. Phys. Lett. 98, 162502 (2011).

[22] Y.-T. Cui, G. Finocchio, C. Wang, J. A. Katine, R. A. Buhrman, and D. C. Ralph, Single-Shot Time-Domain Studies of Spin-Torque-Driven Switching in Magnetic Tunnel Junctions, Phys. Rev. Lett. 104, 097201 (2010).

[23] R. F. L. Evans, W. J. Fan, P. Chureemart, T. A. Ostler, M. O. A. Ellis, and R. W. Chantrell, Atomistic spin model simulations of magnetic nanomaterials, J. Phys.: Condens. Matter 26, 103202 (2014).

[24] S. Ikeda, K. Miura, H. Yamamoto, K. Mizunuma, H. D. Gan, M. Endo, S. Kanai, J. Hayakawa, F. Matsukura, and H. Ohno, A perpendicular-anisotropy $\mathrm{CoFeB}-\mathrm{MgO}$ magnetic tunnel junction, Nat. Mater. 9, 721 (2010).

[25] H. X. Yang, M. Chshiev, B. Dieny, J. H. Lee, A. Manchon, and K. H. Shin, First-principles investigation of the very large perpendicular magnetic anisotropy at $\mathrm{Fe} \mid \mathrm{MgO}$ and $\mathrm{Co} \mid \mathrm{MgO}$ interfaces, Phys. Rev. B 84, 054401 (2011).

[26] Y. Lu, C. Deranlot, A. Vaurès, F. Petroff, J.-M. George, Y. Zheng, and D. Demailles, Effects of a thin $\mathrm{Mg}$ layer on the structural and magnetoresistance properties of $\mathrm{CoFeB} / \mathrm{MgO} / \mathrm{CoFeB}$ magnetic tunnel junctions, Appl. Phys. Lett. 91, 222504 (2007).

[27] S. Yuasa and D. D. Djayaprawira, Giant tunnel magnetoresistance in magnetic tunnel junctions with a crystalline MgO (001) barrier, J. Phys. D: Appl. Phys. 40, R337 (2007).

[28] S. Le Gall, J. Cucchiara, M. Gottwald, C. Berthelot, C.-H. Lambert, Y. Henry, D. Bedau, D. B. Gopman, H. Liu, A. D. Kent, J. Z. Sun, W. Lin, D. Ravelosona, J. A. Katine, Eric E. Fullerton, and S. Mangin, State diagram of nanopillar spin-valve with perpendicular magnetic anisotropy, Phys. Rev. B 86, 014419 (2012).

[29] S. Zhang, P. M. Levy, A. C. Marley, and S. S. P. Parkin, Quenching of Magnetoresistance by Hot Electrons in Magnetic Tunnel Junctions, Phys. Rev. Lett. 79, 3744 (1997).

[30] S.-C. Oh, S.-Y. Park, A. Manchon, M. Chshiev, J.-H. Han, H.-W. Lee, J.-E. Lee, K.-T. Nam, Y. Jo, Y.-C. Kong, B. Dieny, and K.-J. Lee, Bias-voltage dependence of perpendicular spin-transfer torque in asymmetric $\mathrm{MgO}$-based magnetic tunnel junctions, Nat. Phys. 5, 898 (2009).

[31] J. Z. Sun and D. C. Ralph, Magnetoresistance and spintransfer torque in magnetic tunnel junctions, J. Magn. Magn. Mater. 320, 1227 (2008).

[32] K.-J. Lee, A. Deac, O. Redon, J.-P. Nozières, and B. Dieny, Excitations of incoherent spin-waves due to spin-transfer torque, Nat. Mater. 3, 877 (2004).

[33] S. Mangin, Y. Henry, D. Ravelosona, J. A. Katine, and Eric E. Fullerton, Reducing the critical current for spintransfer switching of perpendicularly magnetized nanomagnets, Appl. Phys. Lett. 94, 012502 (2009).

[34] H. Liu, D. Bedau, J. Z. Sun, S. Mangin, E. E. Fullerton, J. A. Katine, and A. D. Kent, Dynamics of spin torque switching in all-perpendicular spin valve nanopillars, J. Magn. Magn. Mater. 358-359, 233 (2014).

[35] J. C. Sankey, Y.-T. Cui, J. Z. Sun, John C. Slonczewski, R. A. Buhrman, and Daniel C. Ralph, Measurement of the spin-transfer-torque vector in magnetic tunnel junctions, Nat. Phys. 4, 67 (2008).

[36] H. Kubota, A. Fukushima, K. Yakushiji, T. Nagahama, S. Yuasa, K. Ando, H. Maehara, Y. Nagamine, K. Tsunekawa, D. D. Djayaprawira, N. Watanabe, and Y. Suzuki, Quantitative measurement of voltage dependence of spin-transfer torque in $\mathrm{MgO}$-based magnetic tunnel junctions, Nat. Phys. 4, 37 (2008). 